\title{
Real-world data on the clinicopathological traits and outcomes of hospitalized liver hemangioma patients: a multicenter study
}

\author{
Tengqian Tang ${ }^{1 \#}$, Xishu Wang ${ }^{1 \#}$, Yilei $\mathrm{Mao}^{2}$, Jing $\mathrm{Li}^{3}$, Tianfu Wen ${ }^{4}$, Weidong Jia ${ }^{5}$, Yongjun Chen ${ }^{6}$, \\ Tao Peng ${ }^{7}$, Lingxiao Liu ${ }^{8}$, Ruifang Fan ${ }^{9}$, Kuansheng $\mathrm{Ma}^{1}$, Feng Xia ${ }^{1}$ \\ ${ }^{1}$ The Institute of Hepatobiliary Surgery, Southwest Hospital, Army Medical University, Chongqing, China; ${ }^{2}$ Department of Liver Surgery, Peking \\ Union Medical College (PUMC) Hospital, PUMC and Chinese Academy of Medical Sciences, Beijing, China; ${ }^{3}$ The Institute of Hepatobiliary \\ Surgery, Xinqiao Hospital, Army Medical University, Chongqing, China; ${ }^{4}$ Department of Liver Surgery \& Liver Transplantation Center, West China \\ Hospital of Sichuan University, Chengdu, China; ${ }^{5}$ Department of Hepatobiliary Surgery, Anhui Provincial Hospital, Hefei, China; ${ }^{6}$ Department \\ of Surgery, Ruijin Hospital, Shanghai Jiao Tong University School of Medicine, Shanghai, China; ${ }^{7}$ Department of Hepatobiliary Surgery, The \\ First Affiliated Hospital of Guangxi Medical University, Nanning, China; ${ }^{8}$ Department of Interventional Radiology, Zhongshan Hospital, Fudan \\ University; Shanghai Institute of Medical Imaging, Shanghai, China; ${ }^{9}$ Department of Hepatobiliary Surgery, Lanzhou General Hospital of Lanzhou \\ Military Area Command, PLA, Lanzhou, China \\ Contributions: (I) Conception and design: F Xia, T Tang, X Wang; (II) Administrative support: F Xia; (III) Provision of study material or patients: Y \\ Mao, J Li, T Wen, W Jia, Y Chen, T Peng, L Liu, R Fan, K Ma, F Xia; (IV) Collection and assembly of data: T Tang, X Wang; (V) Data analysis and \\ interpretation: T Tang, F Xia; (VI) Manuscript writing: All authors; (VII) Final approval of manuscript: All authors. \\ \#These authors contributed equally to this work. \\ Correspondence to: Feng Xia, MD, PhD. The Institute of Hepatobiliary Surgery, Southwest Hospital, 30 Gaotanyan Street, Sapingba District, \\ Chongqing 400038, China. Email: frankfxia@163.com.
}

Background: There is currently a lack of consensus regarding the clinical features, diagnosis, treatment indications and options, and risk assessment of hepatic hemangioma patients.

Methods: This was a multicenter, real-world study that analyzed a large number of hepatic hemangioma cases in China and included patient data on epidemiology, diagnosis, treatment methods, and outcomes.

Results: A total of 5,143 patients hospitalized for hepatic hemangioma were included, of whom $34.42 \%$ were male and $65.58 \%$ were female. The age distribution was concentrated between 30 and 60 years old, accounting for $87.41 \%$ of the patients. Among the hepatic hemangioma patients, $60.8 \%$ had only one tumor, with the most common pathological type being cavernous hemangioma (96.07\% of cases). The treatment motivations and indications included anxiety, obvious clinical symptoms, rapid tumor growth, unclear diagnoses and acute emergencies. Overall, 41.4\% of the patients were treated for psychological reasons, while $30.59 \%$ were treated because they presented obvious (primarily nonspecific) clinical symptoms. Hepatic resection was the main therapeutic method and was based on various indications. There were a small number of patients with Kasabach-Merritt syndrome, according to its generally recognized definition.

Conclusions: Most patients in this study who were hospitalized for hepatic hemangioma did not meet the indications for requiring treatment. Surveillance is the recommended course of action for definitively diagnosed hepatic hemangioma, and a new classification system is needed to standardize the diagnosis of this condition.

Keywords: Liver hemangioma; real-world data; clinicopathological trait; outcome

Submitted Jun 13, 2020. Accepted for publication Mar 28, 2021.

doi: $10.21037 /$ atm-20-4684

View this article at: http://dx.doi.org/10.21037/atm-20-4684 


\section{Introduction}

Adult hepatic hemangioma is the most common benign tumor of the liver. The detection rate of hepatic hemangioma has recently increased because more people are undergoing routine physical examinations and due to advances in imaging examinations. This has resulted in an increasing number of suspected hepatic hemangioma patients who need to be definitively diagnosed and treated. Patients with hepatic hemangioma often feel stressed and anxious. In recent years, research on the etiology, pathogenesis, clinical and imaging characteristics, diagnosis, and treatment of hepatic hemangioma has made progress globally, but there is still a lack of evidence from highquality clinical trials (1). At present, there is no consensus on the clinical features, diagnosis, treatment indications, risk assessment, or treatment strategies for hepatic hemangioma in the literature. This situation has often led to the misdiagnosis and overtreatment of this condition, which can cause serious physical and emotional harm to patients (2-4). Meanwhile, the lack of a clinical classification scheme for hepatic hemangioma has resulted in the absence of a common basis for academic communication, leading to widely variable diagnostic and treatment measures. Therefore, by analyzing and summarizing multicenter realworld data of hospitalized patients, this study provides evidence on the epidemiological characteristics, diagnosis, and treatment of hepatic hemangioma patients in China. In particular, the indications for treatment, therapies, and hepatic hemangioma outcomes are clarified and summarized to improve the understanding of hepatic hemangioma diagnosis and treatment. We present the following article in accordance with the STROBE reporting checklist (available at http://dx.doi.org/10.21037/atm-20-4684).

\section{Methods}

\section{Patients}

We retrospectively analyzed the clinical data of 5,339 patients who were hospitalized with hepatic hemangioma between January 2008 and January 2018 from 26 medical centers in China. These 26 medical centers are all referral hospitals. Hepatic hemangioma was the main cause or the primary diagnosis for all enrolled inpatients and was based on clinical symptoms, imaging results, and/or cytological or histopathological examination. Outpatient and nonhospitalized patients were not included in the study. Patients were also excluded due to incomplete clinicopathological data or if their hepatic hemangioma was combined with malignant tumors or with an inconsistent pathological diagnosis. After the dataset was cleaned and analyzed, the data of 5,143 patients were ultimately included in the study.

\section{Methodology}

The main purpose of the present study was to perform a detailed analysis of the real-world epidemiological characteristics, diagnosis, and treatment of hepatic hemangioma patients. The recorded data included patient age, sex, symptoms, medical history, hepatitis markers, diagnostic methods, imaging features, laboratory test results, liver function classification, pathological results, surgical indications and treatment methods, as well as postoperative short-term and long-term outcomes after treatment. Recurrence was defined as any of the following occurring within two years of the end of the last treatment: a new hepatic hemangioma appearing in the remanent liver, enlargement of original small lesions, or a gradual enlargement of the tumor after treatment. Raw data from the enrolled patients were collected by an assistant and entered into the liver hemangioma database management system at the Clinical Research Center in our institute.

This clinical study strictly followed the Declaration of Helsinki (as revised in 2013) and the principle of "International Ethical Standards for Biomedical Research Involving Human Beings", which was formulated by the World Health Organization and the International Organization of Medical Science Council. All procedures were approved by the Ethics Committee of Southwest Hospital (No. KY201806) and individual consent for this retrospective analysis was waived. Public reports related to this study should not disclose any identifying patient information.

\section{Statistical analysis}

The liver hemangioma database management system was used to establish a database. The data are expressed as numbers and percentages for categorical variables. Frequency distributions are presented in tabular or graphic form. All analyses were carried out using SPSS version 24.0 for Windows (SPSS Inc., Chicago, IL, USA).

\section{Results}

\section{Epidemiological characteristics}

A total of 5,143 patients hospitalized for hepatic hemangioma 


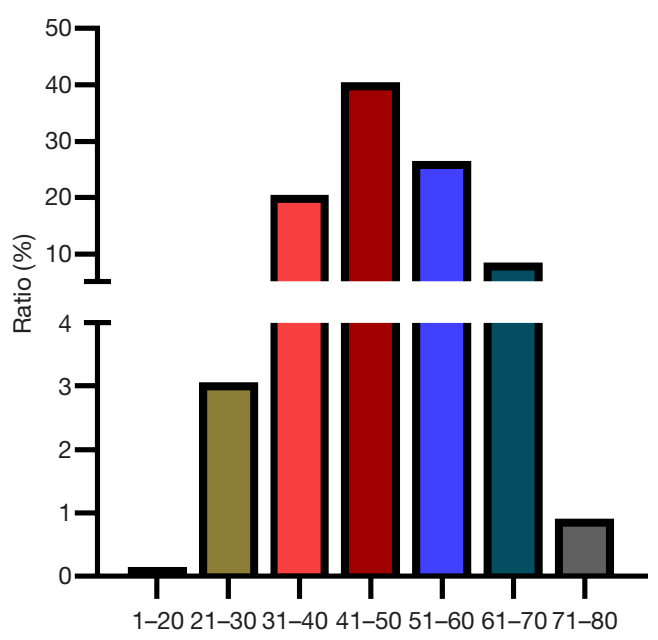

Figure 1 Age distribution of the hepatic hemangioma patients. The age range was concentrated between 30 and 60 years, accounting for $87.41 \%$ of the patients. The age distributions were as follows: 1 to 20 years, $0.14 \% ; 21$ to 30 years, $3.07 \% ; 31$ to 40 years, $20.43 \%$; 41 to 50 years, $40.53 \%$; 51 to 60 years, $26.45 \%$; 61 to 70 years, $8.47 \%$; and 71 to 80 years, $0.91 \%$.

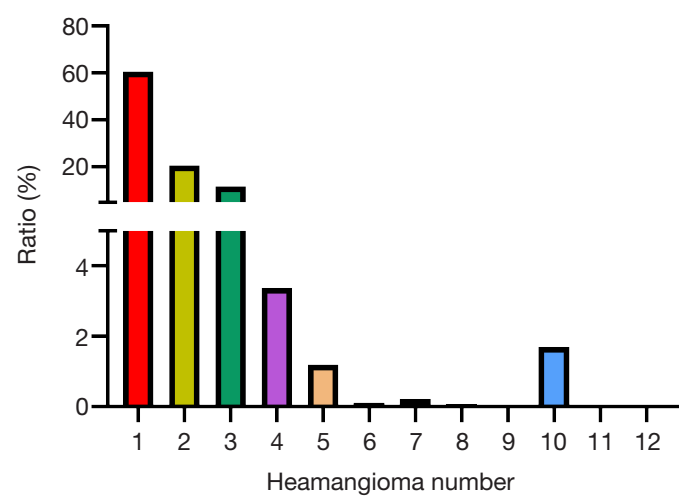

Figure 2 Patient distribution as a function of the number of hemangiomas. Approximately $60.8 \%$ of the patients had only one hepatic hemangioma, $20.69 \%$ had two, and $11.79 \%$ had three.

were included in the study, of whom $34.42 \%$ were male and $65.58 \%$ were female. The age distribution was concentrated between 30 and 60 years old, accounting for $87.41 \%$ of the patients (Figure 1). Patients with hepatitis B and C accounted for $16.25 \%$ and $4.36 \%$, respectively. Liver function was classified according to the Child-Pugh classification: 98.4\% were grade A, $1.6 \%$ were grade $\mathrm{B}$, and there were no cases of grade C. Approximately $2.68 \%$ of the patients showed mild or moderate hepatic sclerosis by imaging examination.

According to the imaging data analysis, $60.8 \%$ of the

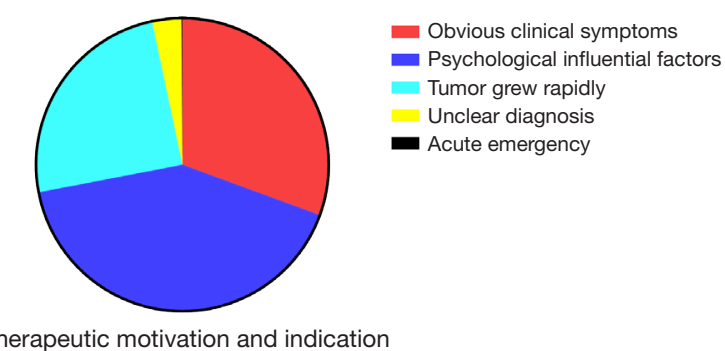

Figure 3 Therapeutic motivation and indications for all cases. Overall, $30.59 \%$ of the patients had obvious clinical symptoms, $41.40 \%$ had influencing psychological factors, $24.69 \%$ had rapidly growing tumors, $3.22 \%$ had an unclear preoperative diagnosis, and $0.10 \%$ required acute emergency treatment.

patients had only one hepatic hemangioma, 20.69\% had two, and $11.79 \%$ had three or more (Figure 2). Regarding the maximum diameter of the hepatic hemangiomas, the tumor diameters in $19.37 \%$ of the patients were $0-5 \mathrm{~cm}$, $61.05 \%$ were $5-10 \mathrm{~cm}, 18.66 \%$ were $10-20 \mathrm{~cm}$, and $0.92 \%$ were greater than $20 \mathrm{~cm}$. Regarding the location of the hepatic hemangiomas, $46.18 \%$ of the patients presented with the tumor in the right hepatic lobe, $25.19 \%$ in the left hepatic lobe, and $28.63 \%$ in both the left and right hepatic lobes. The hemangiomas were found in the subcapsular liver in $25.24 \%$ of the patients and in the intrahepatic parenchyma in the remaining patients $(74.76 \%)$.

Based on the amount of fibrous tissue in the hepatic hemangiomas, these tumors can be classified as sclerosing hemangioma, hemangioendothelioma, cavernous hemangioma and capillary tumor subtypes. Here, cavernous hemangiomas were the most common, accounting for $96.07 \%$. The capillary tumor subtype accounted for $0.70 \%$, the sclerosing hemangioma tumor subtype accounted for $0.55 \%$, and the hemangioendothelioma subtype accounted for $0.48 \%$.

\section{Therapeutic motivation and indications}

The treatment motivations and indications mainly included anxiety, obvious clinical symptoms, rapid tumor growth, unclear diagnosis and acute emergency (Figure 3). Anxiety was the most common treatment motivation, accounting for $41.40 \%$. Overall, $30.59 \%$ of patients were treated because they presented with obvious clinical symptoms. The most common symptoms were nonspecific abdominal pain and distension, and $71.87 \%, 23.93 \%$ and $4.21 \%$ of patients had mild, moderate, or severe symptoms, respectively. Rapid 
Table 1 Clinical classification of hepatic hemangioma

\begin{tabular}{llll}
\hline Classification & Description & $\begin{array}{l}\text { Number of } \\
\text { tumors }\end{array}$ & $\begin{array}{l}\text { Tumor diameter or } \\
\text { sum of diameters or } \\
\text { tumor volume }\end{array}$ \\
\hline la & Single & 1 & $<5 \mathrm{~cm}$ \\
Ib & Single & 1 & $5-10 \mathrm{~cm}$ \\
Ic & Single & 1 & $>10 \mathrm{~cm}$ \\
Ila & Multiple & $2-5$ & $<10 \mathrm{~cm}$ \\
Ilb & Multiple & $2-5$ & $10-20 \mathrm{~cm}$ \\
Ilc & Multiple & $2-5$ & $>20 \mathrm{~cm}$ \\
IIla & Diffuse & $>5$ & $\geq 50 \%$ liver volume \\
IIllb & Diffuse & $>5$ & $>50 \%$ liver volume \\
\hline
\end{tabular}

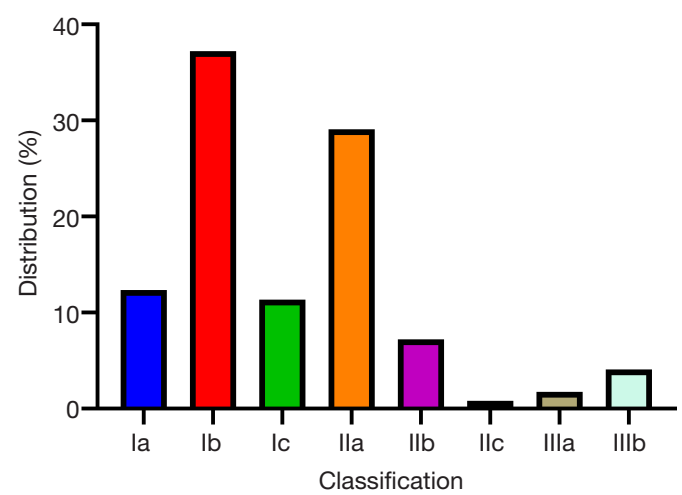

Figure 4 Distribution of patients according to the hepatic hemangioma clinical classification. Most patients were classified as type $\mathrm{Ib}(1,913)$, accounting for $37.2 \%$. The second-largest classification was type IIa $(1,496)$, accounting for $29.1 \%$. Types Ia, Ic, IIb, IIc, IIIa and IIIb accounted for 633 (12.3\%), 581 (11.3\%), 368 (7.2\%), 41 (0.8\%), $90(1.7 \%)$ and 21 (4.1\%) of cases, respectively.

tumor growth was found in $24.69 \%$ of the patients, which is generally designated as an annual increase in tumor diameter of greater than $2 \mathrm{~cm}$. We applied the Chinese clinical classification and subtypes of hepatic hemangioma based on the tumor diameter and tumor number (Table 1). The majority of cases in this real-world study were type IaIIb (Figure 4).

The presence of Kasabach-Merritt syndrome (thrombocytopenia syndrome) necessitates treatment and is defined as giant hemangioma with bleeding tendency and thrombocytopenia. In this study, suspected Kasabach-
Merritt syndrome was found in 19 cases. In these patients, the platelet counts were less than $50 \times 10^{9}$, the hemangioma diameters were greater than $8 \mathrm{~cm}$, and there were no abnormalities in the prothrombin time and hemoglobin. Among these cases, 8 had platelet counts of less than $10 \times 10^{9}, 2$ had platelet counts between $10 \times 10^{9}$ and $20 \times 10^{9}$, 2 had platelet counts between $20 \times 10^{9}$ and $30 \times 10^{9}$, and 7 had platelet counts between $30 \times 10^{9}$ and $50 \times 10^{9}$. None of the patients with Kasabach-Merritt syndrome showed coagulopathy.

\section{Therapy options}

The treatments for hepatic hemangioma included laparotomy (LAP), interventional embolization (IE), laparoscopic resection (LAR), radiofrequency ablation (RFA), microwave ablation (MIA), resection combined with RFA (RE\&RF), liver transplantation (LT) and other treatment methods. Surgical resection was the most frequent treatment method (Figure 5).

\section{Complications}

A total of 757 patients had different degrees of postoperative complications (Table 2, Figure 6). According to the Clavien classification system, the postoperative complications were classified as grade I $(78.70 \%)$, grade II $(18.13 \%)$, grade III (2.03\%), grade IV $(1.10 \%)$ or grade $\mathrm{V}(0.05 \%)$. A total of 2,211 patients were treated for hepatic hemangioma by laparotomic hemangioma resection, of whom 405 patients (18.32\%) experienced postoperative complications. Among those with intraoperative blood loss, $69.75 \%$ lost less than $500 \mathrm{~mL}, 18.07 \%$ lost $500-1,000 \mathrm{~mL}, 11.15 \%$ lost 1,000 $3,000 \mathrm{~mL}, 0.86 \%$ lost 3,000-5,000 mL, 0.06\% lost 5,000$10,000 \mathrm{~mL}$, and $0.12 \%$ lost more than $10,000 \mathrm{~mL}$. A total of 928 patients were treated with laparoscopic hemangioma resection, of whom 269 patients $(28.99 \%)$ experienced postoperative complications. Overall, 320 and 1,338 patients were treated with ablation and IE, respectively, of which $35(10.94 \%)$ and 48 (3.59\%) experienced postoperative complications (Figure 7).

\section{Short- and long-term outcomes}

In terms of the length of stay, $38.11 \%$ of the hepatic hemangioma patients were hospitalized for less than 10 days, $52.84 \%$ for $10-20$ days, $8.01 \%$ for 20-30 days, and $1.05 \%$ for over 30 days. A 3 -month postoperative survey 


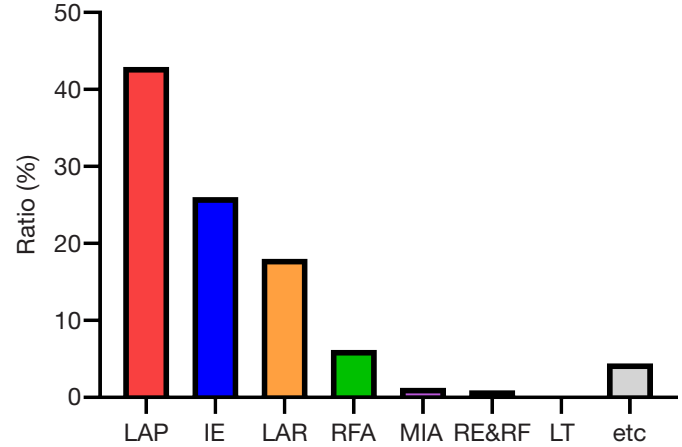

Figure 5 Among the 5,143 patients, 42.98\% received LAP, $26.01 \%$ received IE, $18.05 \%$ received LAR, $6.22 \%$ received RFA, $1.30 \%$ received MIA, $0.94 \%$ received RE\&RF, $0.02 \%$ received LT, and $4.48 \%$ received another treatment method. LAP, laparotomy; IE, interventional embolization; LAR, laparoscopic resection; RFA, radiofrequency ablation; MIA, microwave ablation; RE\&RF, resection combined with RFA; LT, liver transplantation; etc., other treatment.

Table 2 Incidence of postoperative complications (\%) for different treatment methods

\begin{tabular}{lcccc}
\hline $\begin{array}{l}\text { Postoperative } \\
\text { complications }\end{array}$ & $\begin{array}{c}\text { Laparotomic } \\
\text { resection } \\
(\mathrm{n}=405)\end{array}$ & $\begin{array}{c}\text { LAR } \\
(\mathrm{n}=269)\end{array}$ & $\begin{array}{c}\text { Ablation* } \\
(\mathrm{n}=35)\end{array}$ & $\begin{array}{c}\text { IE } \\
(\mathrm{n}=48)\end{array}$ \\
\hline Fever & 39.8 & 20.1 & 2.8 & 4.5 \\
Infection & 26.6 & 11.1 & 1.2 & 0.9 \\
Pleural effusion & 13.3 & 7.1 & 1.5 & 0.5 \\
Ascites & 12.4 & 3.6 & 1.2 & - \\
Hemorrhage & 10.7 & 2.8 & 1.1 & - \\
Bile leakage & 19.9 & 9.4 & 0.8 & - \\
Abdominal pain & 37.9 & 11.8 & 2.6 & 2.2 \\
Liver insufficiency & 8.9 & 2.2 & 1.5 & 0.7 \\
Liver abscess & 2.8 & 0.9 & 2.0 & - \\
Subcutaneous & - & 5.4 & - & - \\
emphysema & & & & \\
\hline
\end{tabular}

*, ablation includes RFA and MIA. LAR, laparoscopic resection; IE, interventional embolization; RFA, radiofrequency ablation; MIA, microwave ablation.

found that $79.44 \%$ of the patients described their symptoms as significantly alleviated, $14.29 \%$ stated that they experienced no significant change, $0.06 \%$ had worsening symptoms, and $6.20 \%$ were unsure about any changes.

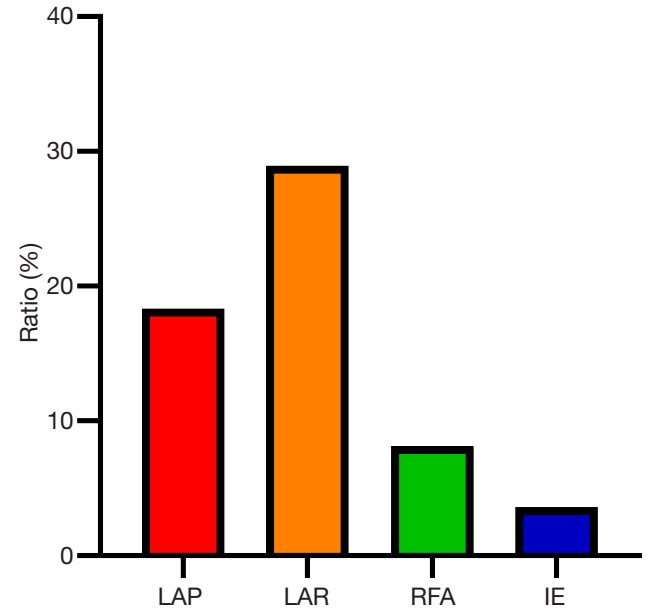

Figure 6 A total of 2,211 patients were treated for hepatic hemangioma with laparotomic hemangioma resection, of whom 405 patients $(18.32 \%)$ experienced postoperative complications. A total of 928 patients were treated with laparoscopic hemangioma resection, of whom 269 patients (28.99\%) experienced postoperative complications. A total of 320 and 1,338 patients were treated with ablation and IE, respectively, of whom 35 (10.94\%) and $48(3.59 \%)$ experienced postoperative complications. LAP, laparotomy; LAR, laparoscopic resection; RFA, radiofrequency ablation; IE, interventional embolization.

In the long term, approximately $3.34 \%$ of the patients experienced recurrence of their hepatic hemangioma after treatment. Forty-one $(1.85 \%)$ of the 2,211 patients treated with laparotomic hemangioma resection experienced hepatic hemangioma recurrence. A total of 928 patients were treated with laparoscopic hemangioma resection, of whom 18 patients (1.94\%) experienced recurrence. Twentyfive patients (7.81\%) experienced recurrence after RFA, and $83(6.20 \%)$ and $5(7.25 \%)$ experienced recurrence after IE and microwave treatment, respectively (Figure 8).

\section{Discussion}

A real-world multicenter study can reveal much more information about a disease than randomized clinical trials owing to the large sample size and full data sets that can be obtained. With the increased understanding of hepatic hemangioma, and especially with the rapid development of imaging technology (5-8), the detection rate and diagnostic accuracy of this disease have improved $(1,9)$. In the present study, the ratio of male to female in-hospital patients with hepatic hemangioma was $1: 1.9$, which is similar to previous 

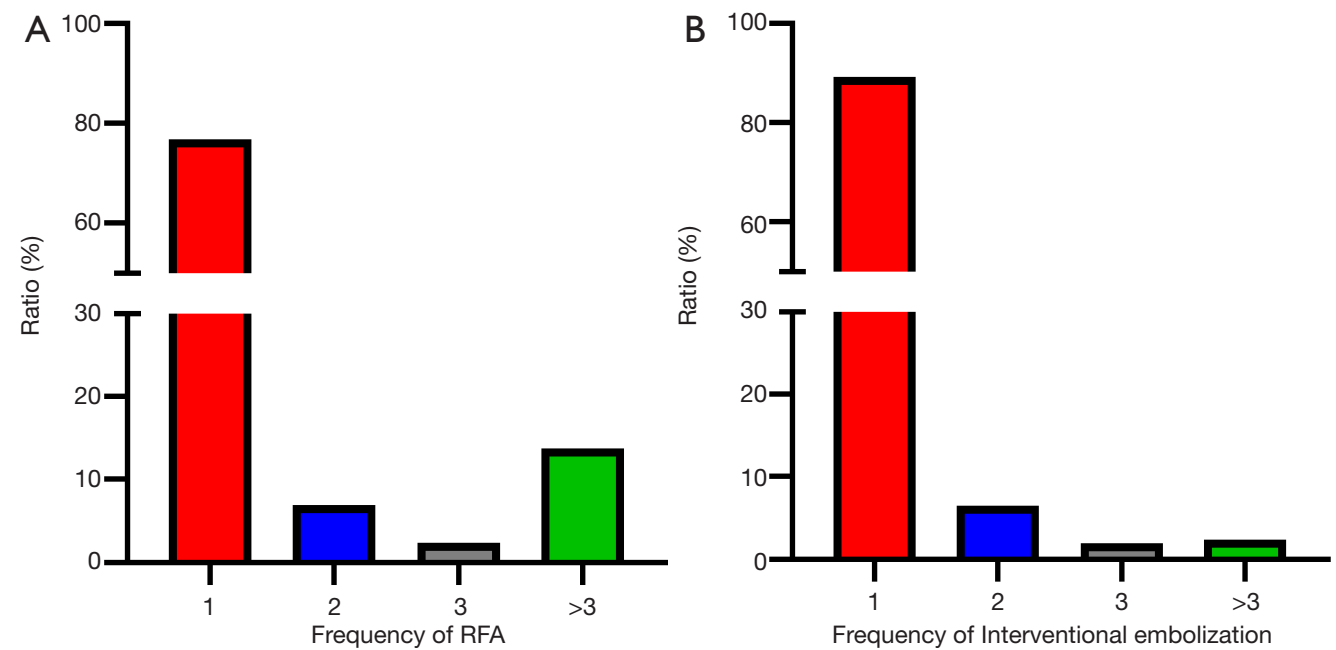

Figure 7 Frequency of RFA and IE were presented. (A) Among the patients treated with RFA, 76.90\% were treated once, 6.90\% were treated twice, $2.41 \%$ were treated 3 times, and $13.79 \%$ were treated more than 3 times. (B) Among the patients treated after IE, $89.19 \%$ were treated once, $6.45 \%$ were treated twice, $1.99 \%$ were treated 3 times, and $2.37 \%$ were treated more than 3 times. RFA, radiofrequency ablation; IE, interventional embolization.

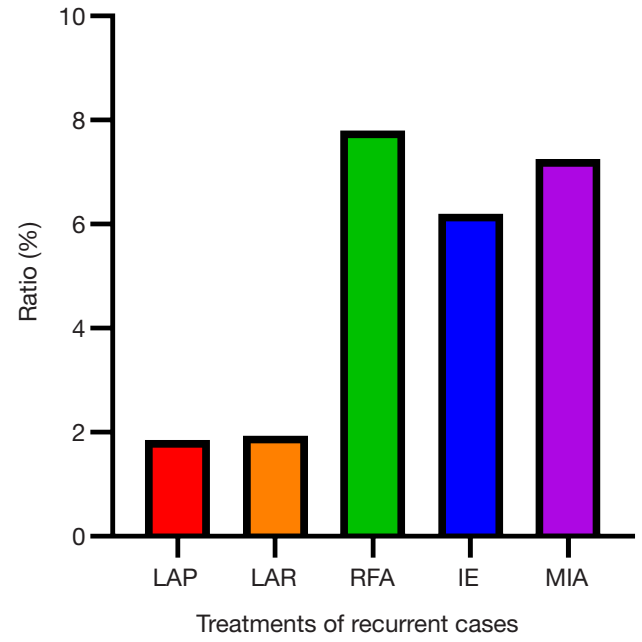

Figure 8 An analysis of recurrent cases by treatment method showed that overall, $3.34 \%$ of the patients experienced hepatic hemangioma recurrence after treatment. Specifically, recurrence occurred in $41(1.85 \%)$ of the 2,211 patients treated with laparotomic hemangioma resection and in 18 (1.94\%) of the 928 patients treated with laparoscopic hemangioma resection. Additionally, 25 patients (7.81\%) experienced recurrence after RFA, and $83(6.20 \%)$ and $5(7.25 \%)$ patients experienced recurrence after IE and microwave treatment, respectively. LAP, laparotomy; LAR, laparoscopic resection; RFA, radiofrequency ablation; IE, interventional embolization; MIA, microwave ablation. reports. The age distribution of patients was concentrated between 30 and 60 years, accounting for $87.41 \%$ of the patients. Approximately $60.8 \%$ of the patients had only one hepatic hemangioma. Previous studies have shown that sex hormones can promote the proliferation, transition and formation of the capillary-like structures of vascular endothelial cells. For instance, pregnancy and oral contraceptives can increase estrogen and progesterone levels, leading to hemangioma growth, which may be related to the higher incidence of this disease in women $(10,11)$. The most common pathological type of hepatic hemangioma was cavernous hemangioma, accounting for $96.07 \%$ of the cases. The morphology of cavernous hemangioma is diverse, and the ratio of regular circle and oval to irregular lesions was approximately $2: 1$. Irregular lesions present with a large flake or lobulated appearance, and the surface is uneven or has a navel concavity (12). Hepatic hemangioma is usually termed cavernous hemangioma, but it has a diverse appearance (13).

It is necessary to determine which types of hepatic hemangioma need to be treated (14). As a benign tumor, most hepatic hemangiomas are asymptomatic and are not typically malignant, and significant liver function abnormalities have not been observed in asymptomatic hepatic hemangioma patients $(6,15,16)$. Surveillance is often recommended for patients presenting with this type of hepatic hemangioma, and this course of action is generally 
accepted worldwide $(14,16)$. In our study, we found that $30.59 \%$ of the inpatients with hepatic hemangioma were treated due to obvious clinical symptoms, and $71.87 \%$ of these patients had mild symptoms. When the tumor diameter is greater than $5 \mathrm{~cm}$, clinical symptoms often arise due to the compression of adjacent tissues and organs. These symptoms mainly present as discomfort or swelling pain in the right rib region. Occasionally, dyspepsia, nausea, and vomiting occur caused by giant hemangioma in the left liver compressing the gastrointestinal tract. There are rare cases of abdominal bleeding from spontaneous or traumatic rupture $(10,11)$.

We found that $20.49 \%$ of the patients experienced little or no change after treatment, and $0.06 \%$ of the patients stated that their health condition deteriorated after treatment. Most of the discomfort experienced by these patients was caused by other digestive tract lesions, such as gastric ulcers, chronic gastroenteritis, chronic cholecystitis and cholangitis or cholelithiasis, and some discomfort was likely caused by psychological factors. Although most clinicians utilize apparent symptoms to guide their hepatic hemangioma treatment decisions (17), it is important to exclude nonspecific manifestations caused by other lesions before conducting treatment.

Spontaneous or traumatic rupture of hepatic hemangioma and Kasabach-Merritt syndrome are absolute indications for treatment because of their fatal consequences $(11,18)$. Other factors, such as cases complicated by obstructive jaundice, portal hypertension, or Budd-Chiari syndrome, are also considered indications for hepatic hemangioma treatment. The mortality of hepatic hemangioma rupture hemorrhage is $35 \%$, which is another absolute indication for surgery $(11,19)$. According to the Medline database, from 1898 to 2010, 97 cases of hepatic hemangioma rupture and hemorrhage were reported, among which 46 were spontaneous rupture and hemorrhage, suggesting that it is extremely rare $(11,19)$. Additionally, KasabachMerritt syndrome has no definition for adult patients, and it has been reported only in individual cases in previous studies. In our multicenter real-world study, there were only a few suspected cases of Kasabach-Merritt syndrome, indicating that this type of hepatic hemangioma is quite rare. Therefore, treatment should be limited to patients with moderate or severe symptoms that are causally related to hepatic hemangiomas and that affect quality of life and patients with serious complications or those who are at significant risk of serious complications.

Some patients experience anxiety and fear because they are worried about a misdiagnosis, malignant transition, tumor growth or spontaneous rupture. The results of this study showed that $41.4 \%$ of the patients underwent hepatic hemangioma treatment for these reasons. There is no consensus on whether psychological factors should be regarded as a surgical indication for hepatic hemangioma because psychological evaluation is complex and difficult. Previous studies have found that few patients experienced relief from their psychological symptoms after the operation, which does carry risk $(11,19)$. Hence, it is necessary for patients to consult a psychologist before deciding to undergo treatment. The symptoms of hepatic hemangioma and the associated anxiety are generally subjective rather than objective indicators. Hence, patients with subjective indications for treatment should be carefully evaluated.

Several previous studies have shown that the growth rate of most hepatic hemangiomas is extremely slow. Approximately $5-35 \%$ of hemangiomas have been shown to gradually enlarge $(11,19)$. When the diameter of the hepatic hemangioma is greater than $10 \mathrm{~cm}$ and continues to grow or increases rapidly within a short time, it may induce symptoms and related complications $(16,20,21)$. In our study, $25 \%$ of patients were treated for progressive hemangioma enlargement. Rapid growth is generally considered to be an annual increase in diameter of greater than $2 \mathrm{~cm}$. Patients whose tumor is initially found large and are at risk of complicated symptoms should receive clinical treatment (19). Most hemangiomas are diagnosed with typical imaging features; however, some suspected hepatic hemangiomas have atypical imaging features and are a concern to clinicians and patients. Therefore, a suspected hemangioma with an uncertain clinical diagnosis is also considered an indication for treatment, especially for patients with a history of hepatitis, cirrhosis, liver cancer, or other malignancies $(22,23)$. Our results indicated that hemangiomas with an unclear diagnosis accounted for $6.3 \%$ to $38.0 \%$ of all treated patients. In recent years, the incidence of malignant tumors has continued to rise; when the diagnosis is not clear, it is recommended that the patient be followed up closely and receive decisive treatment if possible.

There are currently two types of surgical excision, LAP and laparoscopy (23). The excision type is chosen based on the location and diameter of the hepatic hemangioma and the technical proficiency of the hospital (24). Surgical procedures include enucleation of the hemangioma, irregular hepatectomy, segmental or hemihepatectomy, and 
extended hemihepatectomy (4,25). Radiofrequency ablation (RFA) is a minimally invasive and effective treatment option for patients with hepatic hemangioma. In this treatment, the main arterial blood supply area of the hemangioma is destroyed to limit the hemangioma as much as possible and reduce the size of the residual lesion (26,27). Several reports have shown a satisfactory effect of RFA treatment on hepatic hemangioma; the tumor volume appears to shrink, and the patient symptoms are relieved $(26,28)$. Some reports have described the application of MIA in the treatment of hepatic hemangioma in some centers; the treatment principle is similar to that of RFA. Ablation has become the primary minimally invasive treatment option for hepatic hemangioma. IE therapy can effectively occlude small branches of the arterial blood supply, inducing hemangioma fibrosis, preventing tumor growth, shrinking the tumor volume, and improving clinical symptoms. Iodol combined with pingyangmycin or bleomycin is commonly used in IE treatments of the hepatic artery $(29,30)$.

In our study, $14.72 \%$ of patients had different degrees of postoperative complications. Tumor diameter and location, hepatectomy volume, intraoperative blood loss and blood transfusion are risk factors for postoperative complications of hepatic hemangioma, but the surgical risk is mainly related to intraoperative blood loss. The complication rate of ablation and IE for hepatic hemangioma was lower than that of surgical resection. However, the recurrence rate of radiofrequency and IE for hepatic hemangioma was significantly higher than that of surgical resection.

This real-world study collected data from 26 national hospitals as the study sample. However, clinician bias and missing data are inevitable because of the large clinical workload and the difficulty of data collection. As a realworld study, there are no restrictions on the objects of study, and the statistical methods were relatively simple.

\section{Conclusions}

Hemangioma rupture, Kasabach-Merritt syndrome, moderate to severe symptoms, suspected hemangioma with an uncertain clinical diagnosis, and progressive hemangioma should be considered as indications for treatment. Most patients in this study who were hospitalized for hepatic hemangioma did not meet the above indications for treatment. Surveillance is the recommended course of action for definitively diagnosed hepatic hemangioma. A new classification system is needed to standardize the diagnosis of this condition.

\section{Acknowledgments}

This work was supported by The First Affiliated Hospital of Anhui Medical University, Beijing Tongren Hospital, Daping Hospital of the Army Medical University, Fudan University Affiliated Tumor Hospital, Guangxi People's Hospital, The First Affiliated Hospital of Jilin University, Jinan Military General Hospital, The First Affiliated Hospital of Kunming Medical University, the Southern Hospital of Southern Medical University, The First Hospital of Shanxi Medical University, Tianjin, the First Central Hospital, affiliated with Zhongshan Hospital of Xiamen University, Xiangya Second Hospital of Central South University, the Tumor Hospital of Xinjiang Medical University, Yunnan First People's Hospital, and The First Affiliated Hospital of Jilin University. We thank American Journal Experts (AJE) for English language editing.

Funding: This study was supported by the Social Livelihood Project Fund of Chongqing (201706-202005) (Project No. cstc2017shmsA0436) and the National Natural Science Foundation of China (Program No. 81773140).

\section{Footnote}

Reporting Checklist: The authors have completed the STROBE reporting checklist. Available at http://dx.doi. org/10.21037/atm-20-4684

Data Sharing Statement: Available at http://dx.doi. org/10.21037/atm-20-4684

Conflicts of interest: All authors have completed the ICMJE uniform disclosure form (available at http://dx.doi. org/10.21037/atm-20-4684). The authors have no conflicts of interest to declare.

Ethical Statement: The authors are accountable for all aspects of the work in ensuring that questions related to the accuracy or integrity of any part of the work are appropriately investigated and resolved. This clinical study strictly followed the Declaration of Helsinki (as revised in 2013) and the principle of "International Ethical Standards for Biomedical Research Involving Human Beings", which was formulated by the World Health Organization and the International Organization of Medical Science Council. All procedures were approved by the Ethics Committee of Southwest Hospital (No. KY201806) and individual consent for this retrospective analysis was waived. 
Open Access Statement: This is an Open Access article distributed in accordance with the Creative Commons Attribution-NonCommercial-NoDerivs 4.0 International License (CC BY-NC-ND 4.0), which permits the noncommercial replication and distribution of the article with the strict proviso that no changes or edits are made and the original work is properly cited (including links to both the formal publication through the relevant DOI and the license). See: https://creativecommons.org/licenses/by-nc-nd/4.0/.

\section{References}

1. Iacobas I, Phung TL, Adams DM, et al. Guidance document for hepatic hemangioma (infantile and congenital) evaluation and monitoring. J Pediatr 2018;203:294-300.e2.

2. Hoekstra LT, Bieze M, Erdogan D, et al. Management of giant liver hemangiomas: an update. Expert Rev Gastroenterol Hepatol 2013;7:263-8.

3. Hajong R. Giant hepatic hemangioma. Indian J Gastroenterol 2013;32:352.

4. Miura JT, Amini A, Schmocker R, et al. Surgical management of hepatic hemangiomas: a multi-institutional experience. HPB (Oxford) 2014;16:924-8.

5. Maruyama $\mathrm{M}$, Isokawa $\mathrm{O}$, Hoshiyama $\mathrm{K}$, et al. Diagnosis and management of giant hepatic hemangioma: the usefulness of contrast-enhanced ultrasonography. Int J Hepatol 2013;2013:802180.

6. Klotz T, Montoriol PF, Da Ines D, et al. Hepatic haemangioma: common and uncommon imaging features. Diagn Interv Imaging 2013;94:849-59.

7. Vernuccio F, Bruno A, Costanzo V, et al. Comparison of the enhancement pattern of hepatic hemangioma on magnetic resonance imaging performed with Gd-EOBDTPA versus Gd-BOPTA. Curr Probl Diagn Radiol 2020;49:398-403.

8. Tateyama A, Fukukura Y, Takumi K, et al. Gd-EOBDTPA-enhanced magnetic resonance imaging features of hepatic hemangioma compared with enhanced computed tomography. World J Gastroenterol 2012;18:6269-76.

9. Zavras N, Dimopoulou A, Machairas N, et al. Infantile hepatic hemangioma: current state of the art, controversies, and perspectives. Eur J Pediatr 2020;179:1-8.

10. Donati M, Stavrou GA, Donati A, et al. The risk of spontaneous rupture of liver hemangiomas: a critical review of the literature. J Hepatobiliary Pancreat Sci 2011;18:797-805.

11. Gupta S, Agarwal V, Acharya AN. Spontaneous rupture of a giant hepatic hemangioma-report of a case. Indian J Surg 2012;74:434-6.

12. Theodosopoulos T, Dellaportas D, Tsangkas A, et al. Clinicopathological features and management of hepatic vascular tumors. A 20-year experience in a Greek University Hospital. J BUON 2013;18:1026-31.

13. Blumenthal S, Stefanko N, Cossio ML, et al. Multifocal congenital hemangioma: Expanding the pathogenesis of "neonatal hemangiomatosis". Pediatr Dermatol 2019;36:720-2.

14. Toro A, Mahfouz AE, Ardiri A, et al. What is changing in indications and treatment of hepatic hemangiomas. A review. Ann Hepatol 2014;13:327-39.

15. Dong J, Zhang M, Chen JQ, et al. Tumor size is not a criterion for resection during the management of giant hemangioma of the liver. Eur J Gastroenterol Hepatol 2015;27:686-91.

16. Sakamoto Y, Kokudo N, Watadani T, et al. Proposal of size-based surgical indication criteria for liver hemangioma based on a nationwide survey in Japan. J Hepatobiliary Pancreat Sci 2017;24:417-25.

17. Dong W, Qiu B, Xu H, et al. Invasive management of symptomatic hepatic hemangioma. Eur J Gastroenterol Hepatol 2019;31:1079-84.

18. Fujii F, Kimura T, Tanaka N, et al. Hepatic angiosarcoma with Kasabach-Merritt phenomenon: a case report and review of the literature. Ann Hepatol 2018;17:655-60.

19. Di Carlo I, Koshy R, Al Mudares S, et al. Giant cavernous liver hemangiomas: is it the time to change the size categories? Hepatobiliary Pancreat Dis Int 2016;15:21-9.

20. Abdel Wahab M, El Nakeeb A, Ali MA, et al. Surgical management of giant hepatic hemangioma: single center's experience with 144 patients. J Gastrointest Surg 2018;22:849-58.

21. Liu X, Yang Z, Tan H, et al. Characteristics and operative treatment of extremely giant liver hemangioma $>20 \mathrm{~cm}$. Surgery 2017;161:1514-24.

22. Yedibela S, Alibek S, Muller V, et al. Management of hemangioma of the liver: surgical therapy or observation? World J Surg 2013;37:1303-12.

23. Xia F, Lau WY, Qian C, et al. Surgical treatment of giant liver hemangiomas: enucleation with continuous occlusion of hepatic artery proper and intermittent Pringle maneuver. World J Surg 2010;34:2162-7.

24. Veerankutty FH, Rather SA, Yeldho V, et al. Totally laparoscopic resection of an extremely giant hepatic hemangioma. Surg J (N Y) 2019;5:e110-2.

25. Qiu J, Chen S, Wu H. Quality of life can be improved 


\section{Page 10 of 10}

by surgical management of giant hepatic haemangioma with enucleation as the preferred option. HPB (Oxford) 2015;17:490-4.

26. Wen SQ, Wan M, Len KM, et al. Safety and efficacy of laparoscopic radiofrequency ablation for hepatic hemangiomas: a multicenter retrospective study. Ann Hepatol 2018;17:268-73.

27. Sharpe EE 3rd, Dodd GD 3rd. Percutaneous radiofrequency ablation of symptomatic giant hepatic cavernous hemangiomas: report of two cases and review of literature. J Vasc Interv Radiol 2012;23:971-5.

Cite this article as: Tang T, Wang X, Mao Y, Li J, Wen T, Jia W, Chen Y, Peng T, Liu L, Fan R, Ma K, Xia F. Realworld data on the clinicopathological traits and outcomes of hospitalized liver hemangioma patients: a multicenter study. Ann Transl Med 2021;9(13):1067. doi: 10.21037/atm-20-4684

\section{Tang et al. Real-world data on hospitalized liver hemangioma}

28. Zou H, Yan J, Wu YX, et al. The new technology of enhanced radiofrequency ablation is safe and effective for treating giant hepatic hemangioma. Zhonghua Gan Zang Bing Za Zhi 2012;20:261-5.

29. Liu X, Yang Z, Tan H, et al. Long-term result of transcatheter arterial embolization for liver hemangioma. Medicine (Baltimore) 2017;96:e9029.

30. Sun JH, Nie CH, Zhang YL, et al. Transcatheter arterial embolization alone for giant hepatic hemangioma. PLoS One 2015;10:e135158. 